\title{
Caspase-1-mediated regulation of fibrogenesis in diet-induced steatohepatitis
}

\author{
Laura J Dixon ${ }^{1,2}$, Michael Berk²,3, Samjhana Thapaliya ${ }^{2,3}$, Bettina G Papouchado ${ }^{4}$ and Ariel E Feldstein ${ }^{2,3,5}$
}

Non-alcoholic steatohepatitis (NASH) is typically associated with pro-apoptotic caspase activation. A potential role for proinflammatory caspases remains incompletely understood. Our aims were to examine a potential role of caspase- 1 in the development of liver damage and fibrosis in NASH. C57BL/6 wild type (WT) developed marked steatohepatitis, activation, fibrosis and increased hepatic caspase- 1 and interleukin- $1 \beta$ expression when placed on the methionine- and cholinedeficient (MCD) diet. Marked caspase-1 activation was detected in the liver of MCD-fed mice. Hepatocyte and nonparenchymal fractionation of the livers further demonstrated that caspase-1 activation after MCD feeding was mainly localized to non-parenchymal cells. Caspase-1-knockout $\left(\operatorname{Casp~}^{-1-}\right)$ mice on the MCD diet showed marked reduction in mRNA expression of genes involved in inflammation and fibrogenesis (tumor necrosis factor- $\alpha$ was 7.6-fold greater in WT vs Casp $1^{-1-}$ MCD-fed mice; F4/80 was 1.5-fold greater in WT vs Casp $1^{-/-}$MCD-fed mice; $\alpha$-smooth muscle actin was 3.2-fold greater in WT vs Casp 1 ${ }^{-1-}$ MCD-fed mice; collagen 1- $\alpha$ was 7.6-fold greater in WT vs Casp $1^{-1-}$ MCD-fed mice; transforming growth factor- $\beta$ was 2.4 -fold greater in WT vs Casp $1^{-1-}$ MCD-fed mice; cysteine- and glycine-rich protein 2 was 3.2 -fold greater in WT vs Casp 1 $1^{-1-}$ MCD-fed mice). Furthermore, Sirius red staining for hepatic collagen deposition was significantly reduced in Casp $1^{-/-}$MCD-fed mice compared with WT MCD-fed animals. However, serum alanine aminotransferase levels, caspase-3 activity and terminal deoxynucleotidyl transferase dUTP nick-end labeling-positive cells were similar in Casp $1^{-1-}$ and WT mice on the MCD diet. Selective Kupffer cell depletion by clodronate injection markedly suppressed MCD-induced caspase-1 activation and protected mice from fibrogenesis and fibrosis associated with this diet. The conclusion of this study is that it uncovers a novel role for caspase-1 in inflammation and fibrosis during NASH development.

Laboratory Investigation (2012) 92, 713-723; doi:10.1038/labinvest.2012.45; published online 12 March 2012

KEYWORDS: caspases; fibrosis; inflammasome; inflammation; non-alcoholic fatty liver disease; non-alcoholic steatohepatitis

Non-alcoholic fatty liver disease (NAFLD) is currently the most common form of chronic liver disease affecting both adults and children, and is strongly associated with obesity and insulin resistance. ${ }^{1,2}$ One in three adults and one in ten children or adolescents in the United States have hepatic steatosis, a stage within the spectrum of NAFLD, that is characterized by triglyceride accumulation in liver cells and follows a benign non-progressive clinical course. ${ }^{3,4}$ Non-alcoholic steatohepatitis (NASH) is defined as lipid accumulation with evidence of cellular damage, inflammation and different degrees of scarring or fibrosis. ${ }^{5} \mathrm{NASH}$ is a serious condition, as approximately $25 \%$ of these patients progress to cirrhosis and its feared complications of portal hypertension, liver failure and hepatocellular carcinoma. ${ }^{6-8}$ The pathogenesis of NAFLD/NASH, in particular the mechanisms responsible for liver injury and disease progression, remains incompletely understood, but are of significant biomedical importance, as identification of these processes may help to identify novel diagnostic and therapeutic targets for this highly prevalent and potentially serious disease.

Since the original description that caspase activation and hepatocyte apoptosis are characteristic pathological features in the liver of NASH patients, ${ }^{9}$ a growth of data have demonstrated a key role for caspase-dependent cell death in NASH pathogenesis. ${ }^{10-13}$ Caspases are a family of cysteine proteases with unique substrate specificities that have a central role in the apoptotic machinery. ${ }^{14,15}$ They are synthesized as inert zymogens and upon receipt of apoptotic stimuli, cells activate initiator caspases, such as caspase- $1,-2,-8,-9$ and -10

\footnotetext{
${ }^{1}$ Department of Molecular Medicine, Cleveland Clinic Lerner College of Medicine of Case Western University, Cleveland, OH, USA; ${ }^{2}$ Department of Cell Biology, Lerner Cleveland Clinic, Cleveland, OH, USA; ${ }^{3}$ Center for Cardiovascular Diagnostics and Prevention, Cleveland Clinic, Cleveland, OH, USA; ${ }^{4}$ Department of Anatomic Pathology, Cleveland Clinic, Cleveland, OH, USA and ${ }^{5}$ Department of Pediatrics, University of California San Diego (UCSD), San Diego, CA, USA

Correspondence: Dr AE Feldstein, Division of Pediatric Gastroenterology, Hepatology and Nutrition, UCSD, 3020 Children's Way, MC 5030, San Diego, CA 92103-8450, USA.
}

E-mail: afeldstein@ucsd.edu

Received 25 August 2011; revised 23 November 2011; accepted 28 November 2011 
that, in turn, proteolytically cleave and activate effector caspases, including caspase- $3,-6$ and -7 . Caspases have been further categorized as either proinflammatory or proapoptotic, depending upon their participation in these cellular programs. The proinflammatory caspases include caspase- $1,-11$ and -12 in mouse and caspase-1, -4 and -5 in human. ${ }^{16}$ Targeting caspase activity and apoptosis has gained significant attention for developing of novel therapeutic diagnostic strategies for NASH patients. Recent data suggest that pancaspase inhibition protects again diet-induced steatohepatitis in different dietary murine models. ${ }^{17-19}$ These pan-caspase inhibitors, not only inhibit caspase-mediated cellular apoptosis, but also block the caspase-1-dependent processing and activation of various proteins with functions in inflammation and tissue repair during tissue damage. However, the contribution of caspase-1-dependent processes to liver injury and fibrosis remains unclear. In the present study, we examined the occurrence and significance of caspase- 1 activation in NASH.

\section{EXPERIMENTAL PROCEDURES}

\section{Animal Studies}

These experimental protocols were approved by the Institutional Animal Care and Use Committee at the Cleveland Clinic. Male C57BL/6 mice, 20-25g of body weight, were purchased from Jackson Laboratory. C57BL/6 caspase-1knockout $\left(\operatorname{Casp}^{-1-}\right)$ mice (generously provided by Dr Richard Flavell from Yale University, New Haven, CT, USA) were described previously. ${ }^{20,21}$ Mice were placed on a methionine- and choline-deficient (MCD) diet (TD 90262, Teklad Mills, Madison, WI, USA), which has been extensively shown to result in steatosis associated with significant inflammation and progressive fibrosis, pathologically similar to human severe steatohepatitis. ${ }^{22,23}$ In this model, steatosis results from both decreased mitochondrial oxidation of fatty acids and decreased export of fatty acids in the form of very low-density lipoprotein. ${ }^{24}$ Identical groups of animals ( $n=5-7$ in each group) received a standard diet consisting of $5 \%$ fat (TD 2918, Teklad Mills) to act as controls (CTL). Total body weight was measured at $0,1,3$ and 6 weeks. Animals in each group were killed after 6 weeks on respective diets.

In selective studies, C57BL/6 male mice of $20-25 \mathrm{~g}$ in weight were placed on the MCD diet for the intravenous injection of liposomes encapsulating phosphate vehicle (PBS) or clodronate (CLOD; $n=3-7$ in each group). After 5 weeks of MCD diet, animals were injected twice 5 days apart with $0.1 \mathrm{ml}$ per $10 \mathrm{~g}$ of body weight of a $1 \mathrm{mg} / \mathrm{ml}$ suspension of liposomes as previously described. ${ }^{25}$ Animals in each group were killed after 6 weeks on respective treatments.

\section{Cell Lines and Culture}

Primary mouse hepatocytes and total non-parenchymal cells were isolated from C57BL/6 mice on a 6-week MCD diet. The mice were anesthetized and the livers were perfused with warm oxygenated Hanks (-) with $1 \mathrm{mM}$ EGTA and $10 \mathrm{mM}$ HEPES, followed by Williams E media containing $10 \mathrm{mg}$ collagenase per mouse. Hepatocytes were collected after centrifugation, and resulting cell suspensions were used to collect total non-parenchymal cells with a Percoll gradient centrifugation. Caspase- 1 and interleukin (IL)- $1 \beta$ expression was determined by western blot analysis as detailed below.

\section{Histopathology, Immunostaining and Serum Assays}

Blood samples and liver tissue were collected under deep anesthesia after a 5-h fast as previously described in detail. ${ }^{26}$ Liver tissue was fixed in 10\% formalin and embedded in Tissue Path (Fisher Scientific, Pittsburgh, PA, USA). Hematoxylin and eosin (H\&E) as well as Oil red O-stained liver specimens were evaluated by light microscopy. Liver triglyceride determinations were performed using a specific kit following manufacturer's instructions (Pointe Scientific). Serum alanine aminotransferase (ALT) determinations were performed using a commercial kit (Sigma Diagnostics). Individual features, including degree of steatosis, inflammation and ballooning, were assessed in the MCD and CTL-fed animals by an experienced pathologist (BGP) in a blinded fashion. Steatosis, inflammation and ballooning were scored on the basis of the NAFLD activity score. ${ }^{27}$

\section{Assessment of Hepatic Caspase-1 Activation and Cellular Localization}

Caspase- 1 activity was determined using $200 \mu$ g whole-liver protein with the Caspase-1 Fluorometric Assay Kit (cat. ab39412) purchased from Abcam. Immunohistochemistry for caspase-1 was performed using paraffin-embedded liver tissue using standard $\mathrm{DAB}$ technique. The following primary antibodies were used: rabbit anti-Caspase-1 (cat. 06-503 dilution 1:80) purchased from Millipore.

\section{Apoptosis Assessment}

Tissue sections $(4 \mu \mathrm{m})$ were prepared, and terminal deoxynucleotidyl transferase dUTP nick-end labeling (TUNEL) assay was performed following manufacturer's instructions (in situ cell death detection kit; Roche Molecular Biochemicals, Mannheim, Germany). Caspase activation was quantified by immunostaining for active caspase-3, using a cleaved caspase-3 antibody (Cell Signaling). Hepatocyte apoptosis in liver sections was quantitated by counting the number of TUNEL-positive or active caspase-3-positive cells in five random microscopic fields $(\times 40)$, as previously described. ${ }^{12}$

\section{Determination of Liver Fibrosis}

Liver fibrosis was quantified with Sirius Red. Direct Red 80 and Fast Green FCF (color index 42053) were provided by Sigma-Aldrich. Liver sections were incubated in the dark for $2 \mathrm{~h}$ at room temperature with an aqueous solution of saturated picric acid containing 0.1\% Fast Green FCF and 0.1\% Direct Red. ${ }^{26}$ Stained slides were washed slowly under running distilled water for $6 \mathrm{~min}$, dehydrated $(3 \mathrm{~min}$ for each 
a

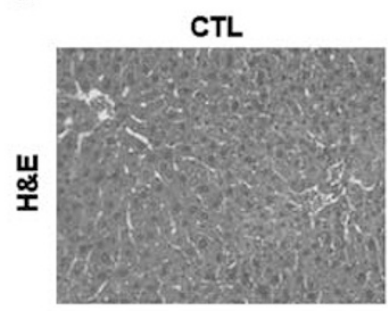

c

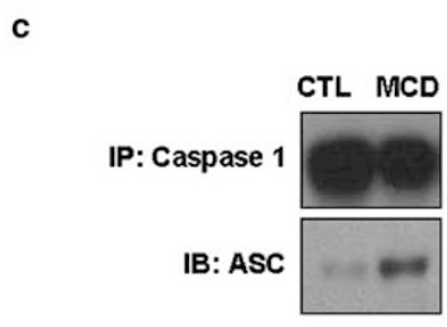

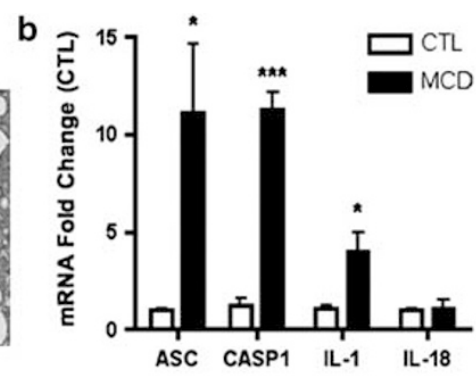

d
*
MCD

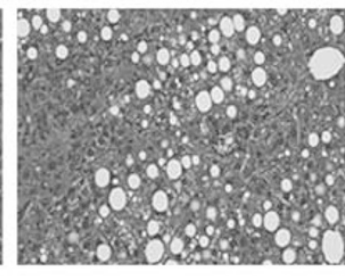

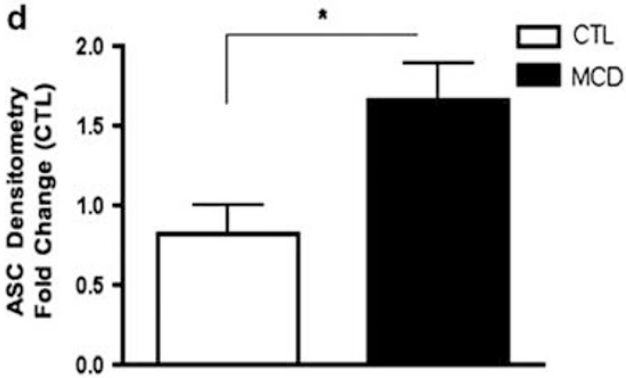
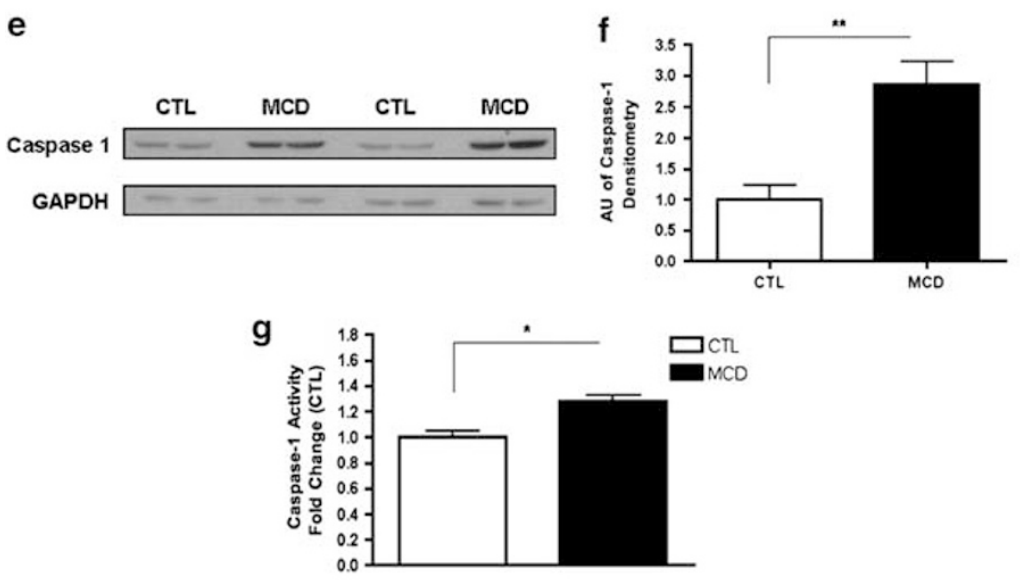
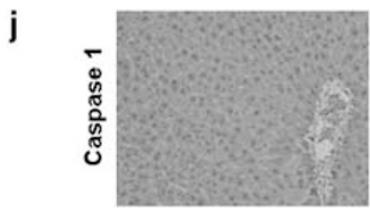

k

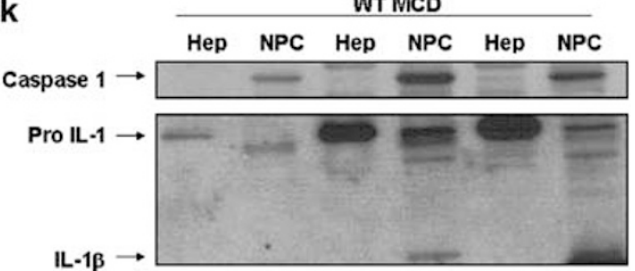

$\mathrm{IL}-1 \beta \longrightarrow$

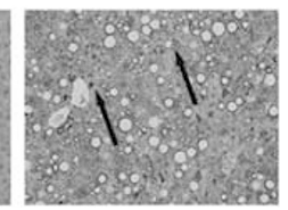

WT MCD h CTL MCD

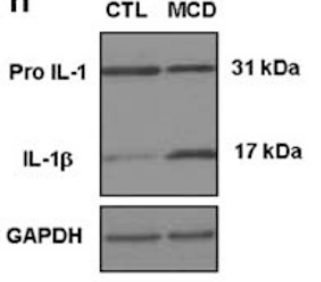

i

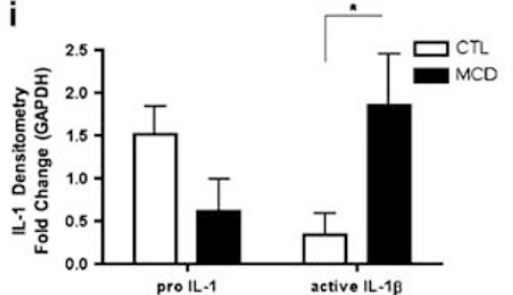

Figure 1 Hepatic caspase-1 activation during diet-induced steatohepatitis. (a) C57BL/6 mice were placed on a methionine- and choline-deficient (MCD) diet or a control (CTL) diet for 6 weeks ( $n=5-7$ in each group). Representative microphotographs of hematoxylin and eosin (H\&E) staining from mice on the two groups $(\times 40)$. (b) RT-PCR analysis of inflammasome components anti-apoptosis-associated speck-like protein containing a caspase recruitment domain (ASC), NACHT, LRR and PYD domains-containing protein 3 (NALP3), caspase-1 (CASP1), interleukin-1 (IL)-1 and IL-18 in liver wild-type (WT) mice ( $n=4$ in each group). (c) Western blot of ASC on caspase-1-immunoprecipitated whole-liver lysates in WT MCD-fed mice compared with WT CTL-fed mice and (d) densitometric analysis of ASC $(n=4)$. (e) Western blot of caspase-1 on whole-liver lysates in WT MCD-fed mice compared with WT CTL-fed mice, and (f) corresponding densitometric analysis to GAPDH $(n=4)$. (g) Caspase-1 activity assay was performed on whole-liver lysates of WT MCD-fed mice and compared with CTL-fed mice ( $n=9$ in each group). (h) Western blots of IL-1 display pro IL-1 processing into active IL-1 $\beta$ in whole-liver lysates of MCD-fed mice compared with CTL-fed mice and corresponding (i) densitometric analysis to GAPDH. (j) Representative microphotograph of caspase-1 immunohistochemistry in paraffin-embedded liver sections of MCD-fed mice compared with CTL-fed animals (magnification $\times 40)$. (k) Western blot of caspase-1 and IL-1 was performed on cell lysates from isolated primary hepatocytes and total non-parenchymal cells from MCD-fed animals. Results are expressed as mean \pm s.e.m. ${ }^{\star} P<0.05,{ }^{* * P}<0.01,{ }^{* * *} P<0.001$, compared with CTLs.

step), mounted, and examined by light microscopy. Redstained collagen fibers were quantitated by digital image analysis, excluding blood vessels.

\section{Immunoblot and Immunoprecipitation Analysis}

Total liver protein $(500 \mu \mathrm{g})$ was immunoprecipitated with $5 \mu \mathrm{g}$ rabbit anti-caspase-1 antibody (cat. 06-503) purchased 
from Millipore. Immunoblot analysis was performed using $30-40 \mu \mathrm{g}$ whole-liver lysate, $20 \mu \mathrm{g}$ hepatocyte or non-parenchymal fraction, or immunoprecipitated lysate. Wholeliver lysate and hepatocyte/non-parenchymal samples were resolved by $12-15 \%$ SDS-PAGE, and immunoprecipitated liver lysate samples were resolved by a $4-20 \%$ gradient gel (cat. EC60285BOX) purchased from Invitrogen, transferred to nitrocellulose membrane, and blotted with appropriate primary antibodies. The membrane was incubated with peroxidase-conjugated secondary antibody (dilution 1:10 000 (BIOSOURCE International, Camarillo, CA, USA), and the bound antibody was visualized using a chemiluminescent substrate (ECL, Amersham Biosciences) and Kodak X-OMAT film (Eastman Kodak, Rochester, NY, USA). The following primary antibodies were used: rabbit anti-Caspase-1 (cat. 06503 dilution 1:80) purchased from Millipore, rabbit antiIL1 $\beta$ (cat. Ab9722, dilution 1:5000) purchased from Abcam; rabbit anti-apoptosis-associated speck-like protein containing a caspase recruitment domain (ASC; cat. ab64808 dilution 1:1000) purchased from Abcam; rabbit anti- $\alpha$ smooth muscle actin (SMA; cat. ab5694 dilution 1:500) purchased from Abcam.

\section{Real-Time PCR}

Total RNA was isolated from liver tissue using RNeasy Tissue Mini kit (Qiagen, Valencia, CA, USA). The reverse transcript (the cDNA) was synthesized from $1 \mu \mathrm{g}$ of total RNA using the iScript cDNA Synthesis kit (Bio-Rad). Real-time PCR quantification was performed. Briefly, $25 \mu \mathrm{l}$ of reaction mix contained: cDNA, Syber Green buffer, Gold Taq polymerase, dNTPs and primers at final concentrations of $200 \mu \mathrm{m}$. The sequences of the primers used for quantitative PCR were as follows: $\alpha$ SMA $5^{\prime}$-GTC CCA GAC ATC AGG GAG TAA- $3^{\prime}$ and $5^{\prime}$-TCG GAT ACT TCA GCG TCA GGA-3'; COL1A1 $5^{\prime}$-CAA GAA CAG CAA CGA GTA CCG- $3^{\prime}$ and $5^{\prime}$-GTC ACT GGT CAA CTC CAG CAC- $3^{\prime}$; transforming growth factor- $\beta$ $\left(\right.$ TGF $\beta$ ) $5^{\prime}$-CTCCCGTGGCTTCTAGTGC-3' and $5^{\prime}$-GCCTTA GTTTGGACAGGATCTG-3' ${ }^{\prime}$; tumor necrosis factor- $\alpha$ (TNF $\left.\alpha\right)$ $5^{\prime}$-CCC TCA CAC TCA GAT CAT CTT CT- $3^{\prime}$ and $5^{\prime}$-GCT ACG ACG TGG GCT ACA G-3'; F4/80 5'-CCCCAGTGT CCTTACAGAGTG- $3^{\prime}$ and $5^{\prime}$-GTGCCCAGAGTGGATGTCT$3^{\prime}$; CD11c 5'-CTG GAT AGC CTT TCT TCT GCT G- $3^{\prime}$ and $5^{\prime}$-GCA CAC TGT GTC CGA ACT CA- $3^{\prime}$; ASC $5^{\prime}$-CTT GTC AGG GGA TGA ACT CAA AA- $3^{\prime}$ and $5^{\prime}$-GCC ATA CGA CTC CAG ATA GTA GC-3'; Casp1 5'-ACA AGG CAC GGG ACC TAT G-3' and $5^{\prime}$-TCC CAG TCA GTC CTG GAA ATG- $3^{\prime}$;
IL-1 $5^{\prime}$-GCA ACT GTT CCT GAA CTC AAC T- $3^{\prime}$ and $5^{\prime}$-ATC TTT TGG GGT CCG TCA ACT-3'; IL-18 5'-GAC TCT TGC GTC AAC TTC AAG G-3' and $5^{\prime}$-CAG GCT GTC TTT TGT CAA CGA- $3^{\prime}$; cysteine- and glycine-rich protein 2 (CRP2) $5^{\prime}$-GCTACGGAAAG AAGTATGGACC- $3^{\prime}$ and $5^{\prime}$-CTCAGTCAAGTTGTAGACTCC-3'; $18 \mathrm{~S}$ ribosomal RNA 5'-ACG GAA GGG CAC CAC CAG GA- $3^{\prime}$ and $5^{\prime}$-CAC CAC CAC CCA CGG AAT CG-3' was used as an endogenous CTL. RT-PCR was performed in the Mx3000P cycler (Stratagene): $95^{\circ} \mathrm{C}$ for $10 \mathrm{~min}, 40$ cycles of $15 \mathrm{~s}$ at $95^{\circ} \mathrm{C}, 30 \mathrm{~s}$ at $60^{\circ} \mathrm{C}, 30 \mathrm{~s}$ at $72^{\circ} \mathrm{C}$, followed by $1 \mathrm{~min}$ at $95^{\circ} \mathrm{C}, 30 \mathrm{~s}$ at $55^{\circ} \mathrm{C}$ and $30 \mathrm{~s}$ at $95^{\circ} \mathrm{C}$. The fold change over CTL samples was calculated using CT, $\Delta \mathrm{CT}$ and $\Delta \Delta \mathrm{CT}$ values using MxPro software (Stratagene).

\section{Statistical Analysis}

All data were expressed as the mean \pm s.e.m. unless otherwise indicated. Differences between three or more groups were compared by an ANOVA analysis followed by a post-hoc Newman-Keuls test, parametric test or the Kruskall-Wallis nonparametric test. Differences between two groups of normalized data were compared by a two-sided Student's $t$-test. Differences were considered to be statistically significant at $P<0.05$. All statistical analysis was performed using GraphPad Prism 4.0c.

\begin{tabular}{|c|c|c|c|c|}
\hline & \multicolumn{2}{|c|}{ WT } & \multicolumn{2}{|c|}{ Casp $1^{-/-}$} \\
\hline & CTL & $M C D$ & CTL & MCD \\
\hline Steatosis & $0.0 \pm 0.0$ & $2.8 \pm 0.2^{* * *}$ & $0.3 \pm 0.3$ & $3.0 \pm 0.0^{* * *}$ \\
\hline Inflammation & $0.0 \pm 0.0$ & $1.3 \pm 0.2^{* *}$ & $0.3 \pm 0.0$ & $0.3 \pm 0.3^{a}$ \\
\hline Ballooning & $0.0 \pm 0.0$ & $0.7 \pm 0.2^{*}$ & $0.0 \pm 0.0$ & $1.0 \pm 0.0^{*}$ \\
\hline NAS & $0.0 \pm 0.0$ & $4.8 \pm 0.5^{* * *}$ & $0.3 \pm 0.3$ & $4.3 \pm 0.3^{* * *}$ \\
\hline
\end{tabular}

Abbreviations: NAFLD, non-alcoholic fatty liver disease; MCD, methionine- and choline-deficient diet; CTL, controls; NAS, NAFLD activity score.

${ }^{\mathrm{a}}$ Comparison of WT MCD and Casp $1^{-/-}$MCD: $P<0.05$ between WT MCD and Casp ${ }^{-/-}$MCD.

Mean \pm s.e.m.

Comparison of CTL and MCD: ${ }^{*} P<0.05,{ }^{* *} P<0.01,{ }^{* * *}<<0.001$.

Figure 2 Caspase-1 suppression is associated with dissociation between hepatic triglyceride accumulation and inflammatory activity in diet-induced steatohepatitis. (a) Body weight was measured at weeks $0,1,3$ and 6 of wild-type (WT) and caspase-1-knockout (Casp $\left.1^{-/}\right)$mice on a 6-week control (CTL) or methionine- and choline-deficient (MCD) diet ( $n=5-7$ in each group). (b, c) Representative images of hematoxylin and eosin (H\&E) and Oil red O (ORO) staining of liver tissue display the severity of cellular injury, further investigated by: (d) hepatic triglyceride (TG; $n=4-5)$ and (e) serum alanine aminotransferase (ALT) levels $(n=5-6)$. (f) RT-PCR analysis of pro-inflammatory cytokine tumor necrosis factor- $\alpha($ TNF $\alpha ; n=3-4)$, (g) macrophage marker F4/ $80(n=6-8)$ and CD11c $(n=4-5)$ in liver of Casp1 ${ }^{-1-}$ mice compared with WT mice. Results are expressed as mean \pm s.e.m. ${ }^{\star} P<0.05$, ${ }^{\star *} P<0.01$, ${ }_{* * *} P<0.001$, compared with CTLs. 


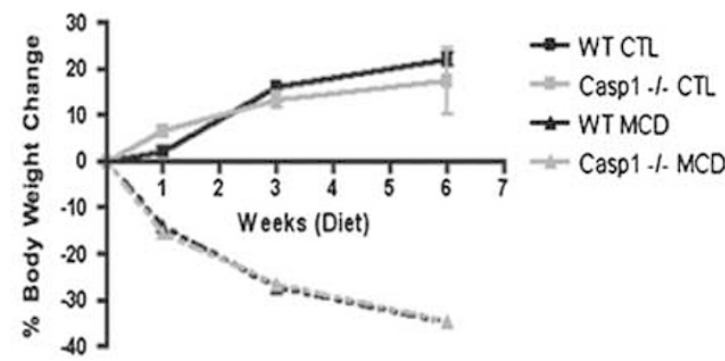

c WT

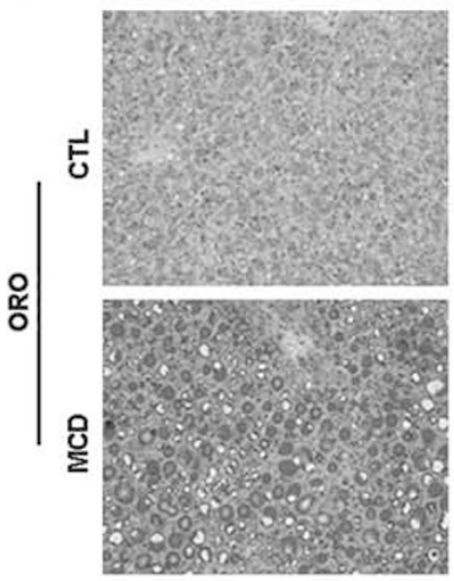

Casp1 -f-

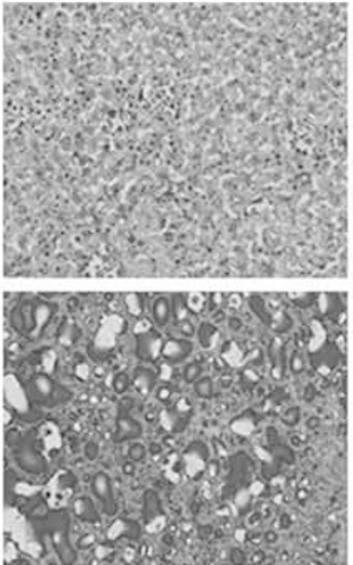

e

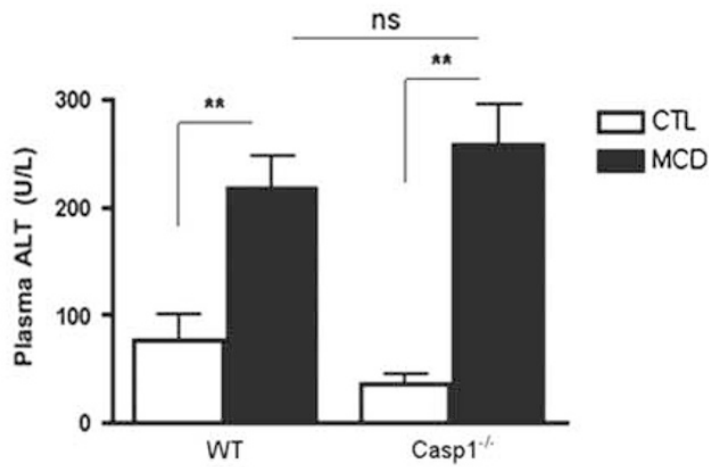

g

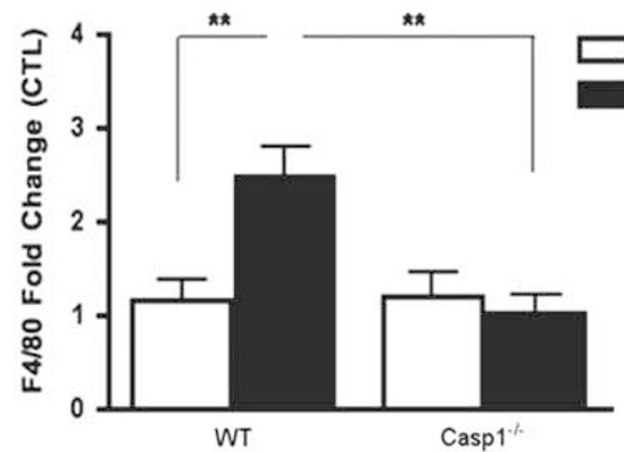

b

WT

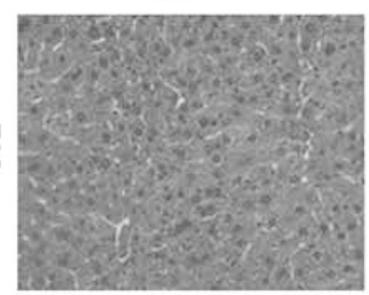

Ш્ఞ

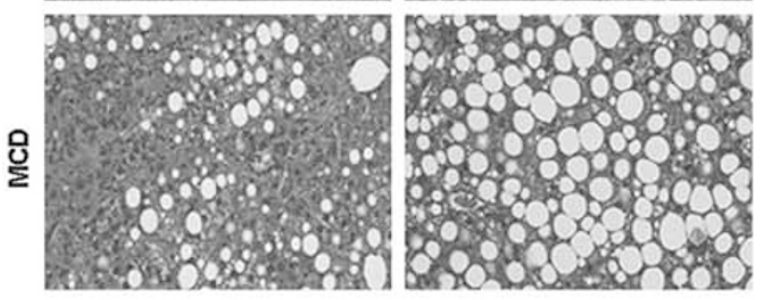

d

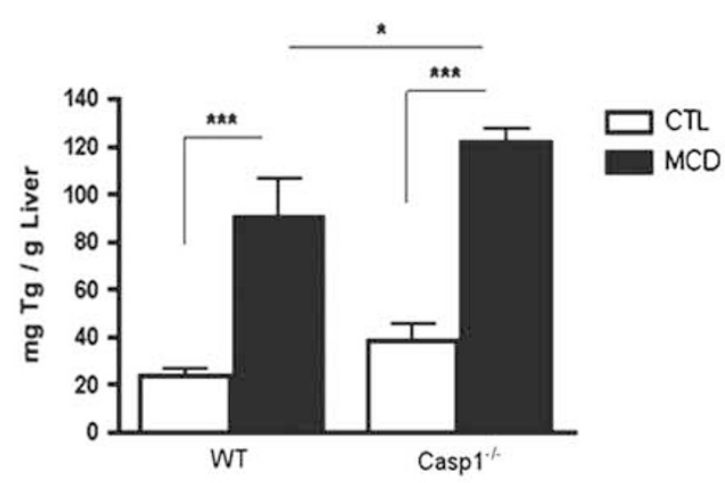

f

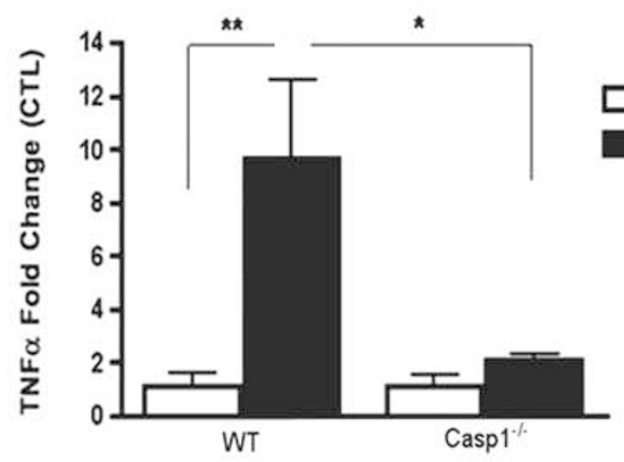

h

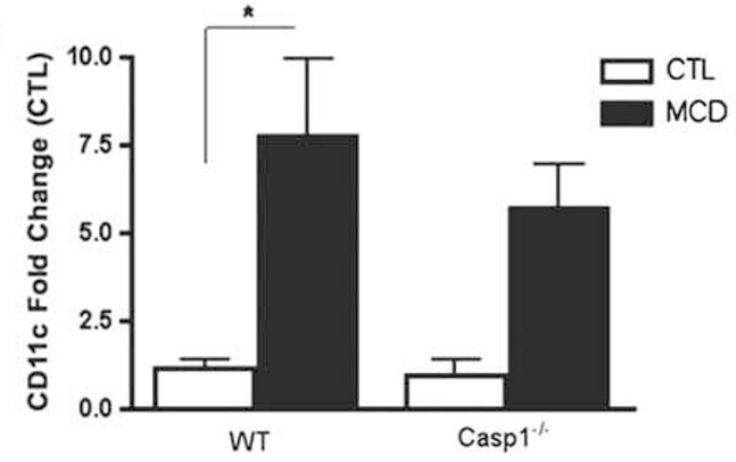




\section{RESULTS}

Hepatic Caspase-1 Activation Is a Prominent Pathological Feature in Experimental NASH

To investigate the role of caspase- 1 activation in the pathogenesis of $\mathrm{NASH}$, we initially placed $\mathrm{C} 57 \mathrm{BL} / 6$ mice on the MCD diet, which has been extensively shown to be associated with progressive fibrosing steatohepatitis, pathologically similar to human severe steatohepatitis. ${ }^{22,23}$ After 6 weeks on the respective diets, we observed significant hepatic fat accumulation induced by MCD feeding (Figure 1a), in conjunction with an increase in histological parameters of liver injury, including hepatic inflammation and hepatocyte ballooning (Figure 1a and Table 1). Expression of caspase-1 was significantly increased in mice on the MCD feeding compared with CTL (Figure 1b). This was accompanied by increased mRNA levels of ASC, a key component of the inflammasome, the protein platform that activates caspase-1 as well as IL-1, but not of IL-18 (Figure 1b). These changes in the livers of mice fed the MCD diet were associated with a marked increased in inflammasome activation (Figure 1c and d), as well as caspase- 1 protein expression and activity levels (Figure 1e-g). Consistent with these data, liver active mature IL-1 $\beta$ protein expression was also significantly increased in MCDfed animals compared with CTL mice (Figure $1 \mathrm{~h}$ and i). Immunohistochemical analysis of liver sections from the two groups of mice showed that caspase-1 was primarily localized to non-parenchymal sinusoidal cells, and to a lesser extent to hepatocytes (Figure 1j). This was further confirmed by fractionation of the liver tissue from MCD-fed animals into hepatocytes and total non-parenchymal cells further demonstrating that caspase- 1 and IL- $1 \beta$ protein expression was markedly enhanced in the non-parenchymal fraction compared with hepatocytes (Figure 1k).

\section{Caspase-1 Suppression Is Associated with Dissociation between Hepatic Triglyceride Accumulation and Inflammatory Activity}

Having established the presence of inflammasome activation and increased caspase- 1 activity in the liver of MCD-fed mice, we next sought to investigate whether caspase-1 participates in $\mathrm{NASH}$ development using $\operatorname{Casp1}^{-1-}$. We first investigated whether $\operatorname{Casp1}^{-1-}$ mice are resistant to hepatic steatosis and inflammation. C57BL/6 wild type (WT), Casp1 knock-out mice were placed on either a MCD diet or CTL diet ( $n=5-7$ in each group) for 6 weeks. WT mice and Casp1 $^{-/-}$mice had similar weight changes on the MCD diet (Figure 2a). However, microscopic examination of H\&E and Oil Red-O staining showed that $\operatorname{Casp}^{-1-}$ mice on the MCD diet developed more significant macrovesicular hepatic steatosis, compared with the WT mice on this diet (Figure 2b and c). Consistently with these results, hepatic triglyceride levels were significantly more elevated in $C a s p 1^{-1-}$ mice compared with WT mice on the MCD diet (Figure 2d). Histological examination of liver samples of other individual features associated with NASH demonstrated a decrease in inflammatory foci, resulting in lower inflammatory activity scores in the Casp1 ${ }^{-/-}$mice on the MCD diet compared with WT mice on this diet (Table 1). However, the total NAFLD activity score was similar in the two groups of mice as mainly as a result of higher degree of steatosis, as well as higher levels of hepatocyte ballooning present in MCD-fed Casp $1^{-1-}$ mice (Table 1). Serum ALT levels were similarly elevated in Casp1 ${ }^{-1-}$ mice and WT mice on the MCD diet compared with animals fed the CTL diet (Figure 2e). We next examined the inflammatory state of the liver at the molecular and cellular level, and found a marked reduction in mRNA levels of TNF $\alpha, F 4 / 80$ and CD11c in the MCD-fed Casp1 $1^{-1-}$ mice compared with the WT animals on the MCD diet (Figure 2f-h).

\section{HSC Activation and Collagen Deposition Induced by the MCD Diet are Decreased by Caspase-1 Suppression Independent of Caspase-3 Activation and Apoptosis}

The findings of a key role of caspase- 1 in hepatocyte injury and inflammatory signaling, two events that have been linked to HSC activation, led us to further examine the role of caspase- 1 in fibrogenesis and fibrosis induced by the MCD diet. Although, as expected, after 6 weeks on the MCD diet, WT animals showed a marked increase in the mRNA expression of genes involved in HSC activation and fibrogenesis, such as TGF $\beta, \alpha \mathrm{SMA}$, COL1A1 and CRP2 (Figure $3 \mathrm{a}-\mathrm{d}$ ). These changes were significantly reduced in the $\operatorname{Casp}^{-1-}$ MCD-fed mice. In addition, $\alpha$ SMA protein expression was increased in WT MCD-fed mice, whereas it was significantly reduced in Casp1 $1^{-/}$MCD-fed mice (Figure 3e and f). More importantly, an almost four-fold increase in collagen deposition, as demonstrated by Sirius red staining of liver tissue coupled to quantitation by digitized image analysis, was present in WT animals on the MCD diet compared with the WT animals on the CTL diet (Figure $3 g$ and $h$ ), whereas these increases in collagen deposition were significantly less prominent in the Casp1 $1^{-/-}$mice, although still higher than in mice on the CTL diet (Figure $3 g$ and $h$ ). We next quantified the amount of hepatocellular cell death present in the various groups of mice. Caspase-3 activation and TUNEL-positive hepatocytes were increased to a similar extent in both WT animals and Casp1 $1^{-/-}$mice on the MCD diet compared with animals on the CTL diet (Figure $4 \mathrm{a}-\mathrm{d}$ ).

Taken together, these observations suggest that during NASH development, caspase-1 activation in hepatocytes has an important role in hepatocellular injury, inflammatory signaling, HSC activation and hepatic fibrosis, independent of caspase- 3 activation and hepatocellular apoptosis.

\section{Selective Kupffer Cell Depletion in MCD-Induced Steatohepatitis Reduces Caspase-1 Activation and Protects Against Fibrogenesis and Fibrosis}

The findings of inflammasome activation and marked increase in caspase- 1 and IL- $1 \beta$, mainly from non-parenchymal cells of the liver, on MCD-fed animals, in conjunction with 
a

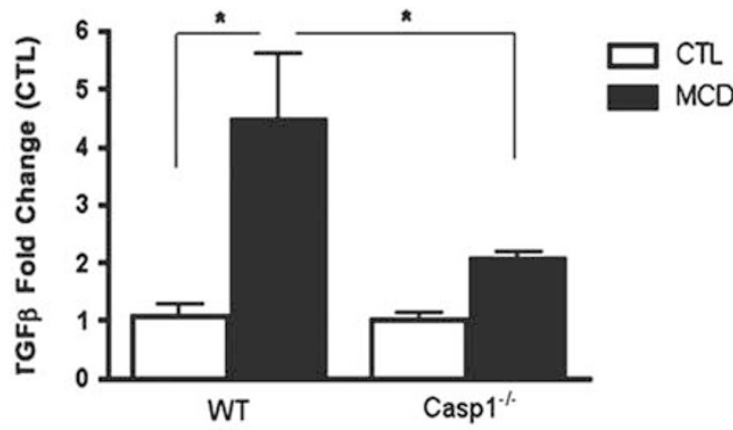

C

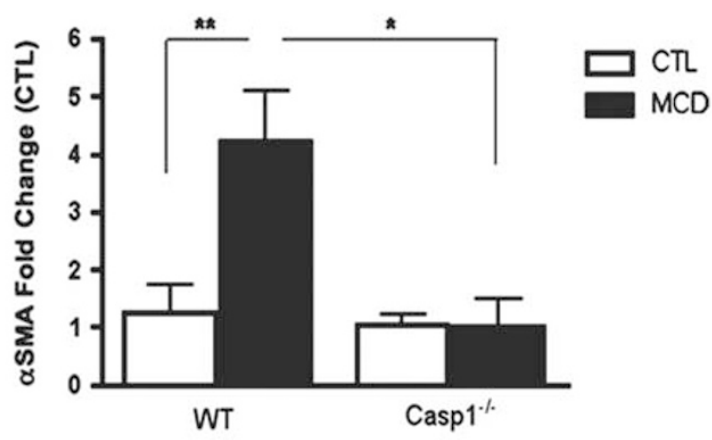

e

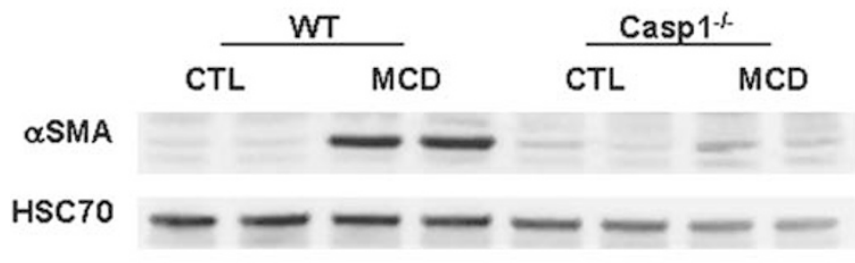

g

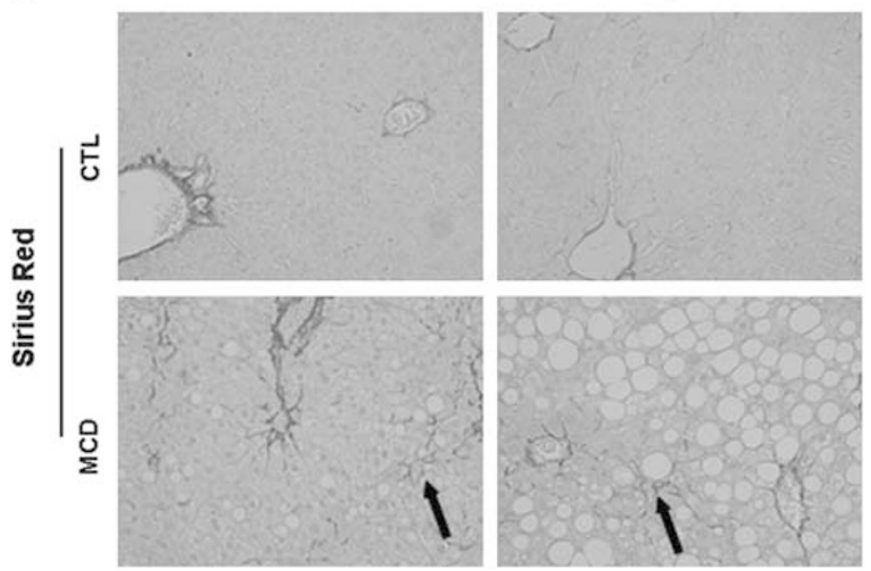

b
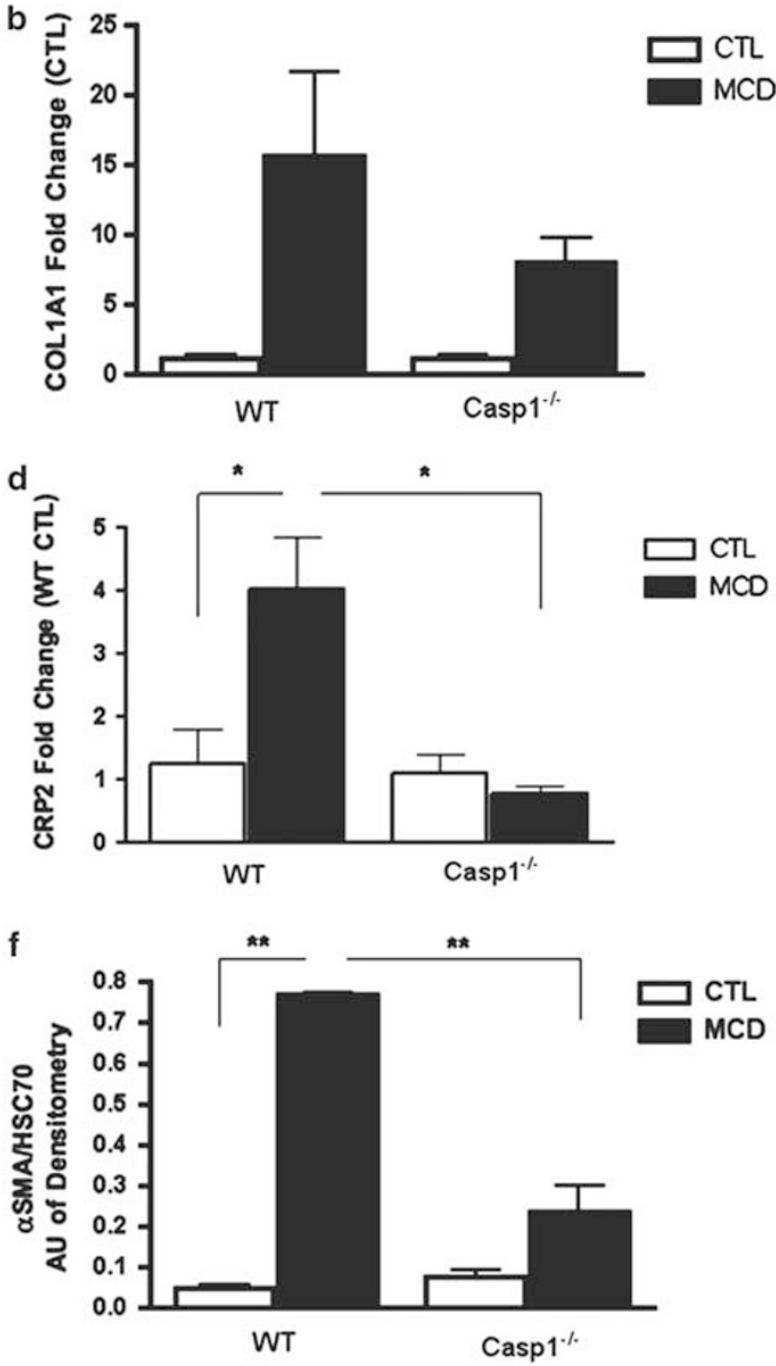

h

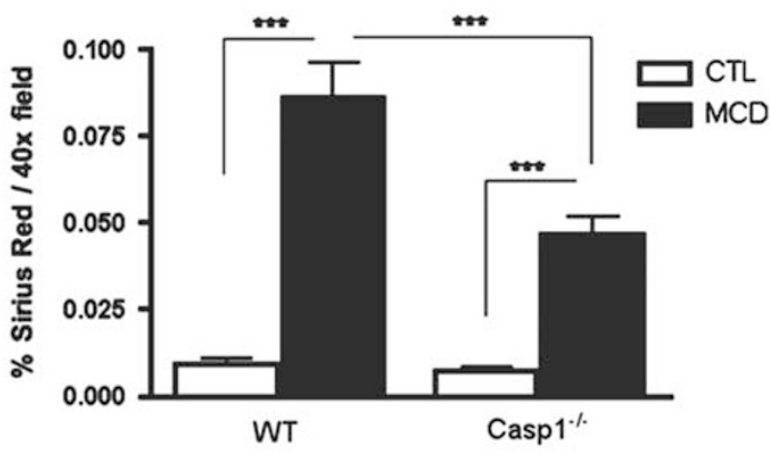

Figure 3 HSC activation and collagen deposition during diet-induced steatohepatitis are markedly decreased in caspase-1-knockout (Casp $\left.1^{-1-}\right)$ mice. (a-d) RT-PCR analysis of HSC activation markers transforming growth factor- $\beta$ (TGF $\beta)$, collagen type-l- $\alpha-1$ (COL1A1), $\alpha$-smooth muscle actin $(\alpha$ SMA), cysteineand glycine-rich protein 2 (CRP2), mRNA expression in liver of Casp $1^{-1-}$ mice compared with wild-type (WT) mice ( $n=5-7$ in each group). (e) Western blot of $\alpha$ SMA on whole-liver lysates in WT and Casp $1^{-1-}$ methionine- and choline-deficient (MCD)-fed mice compared with control (CTL)-fed mice and (f) corresponding densitometric analysis to $\operatorname{HSC70}(n=3)$. (g) Collagen fibers were stained with Sirius red and (h) quantified using the surface area stained per $\times 40$ field area ( $n=5-7$ in each group) excluding blood vessels. Results are expressed as mean \pm s.e.m. ${ }^{\star} P<0.05,{ }^{* *} p<0.01,{ }^{* * \star} P<0.001$, compared with CTLS. 
a

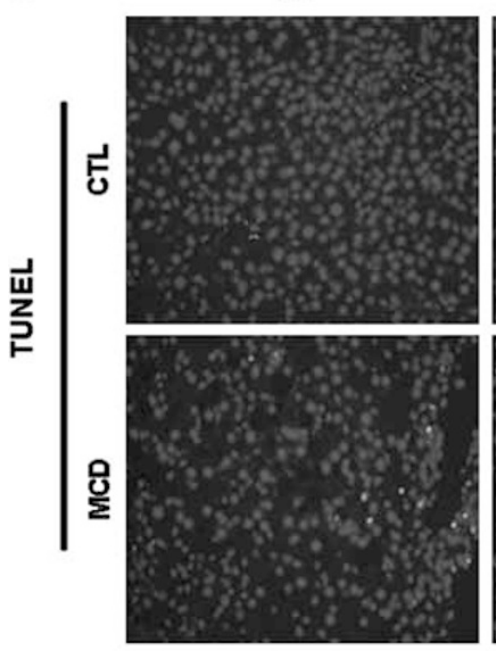

c

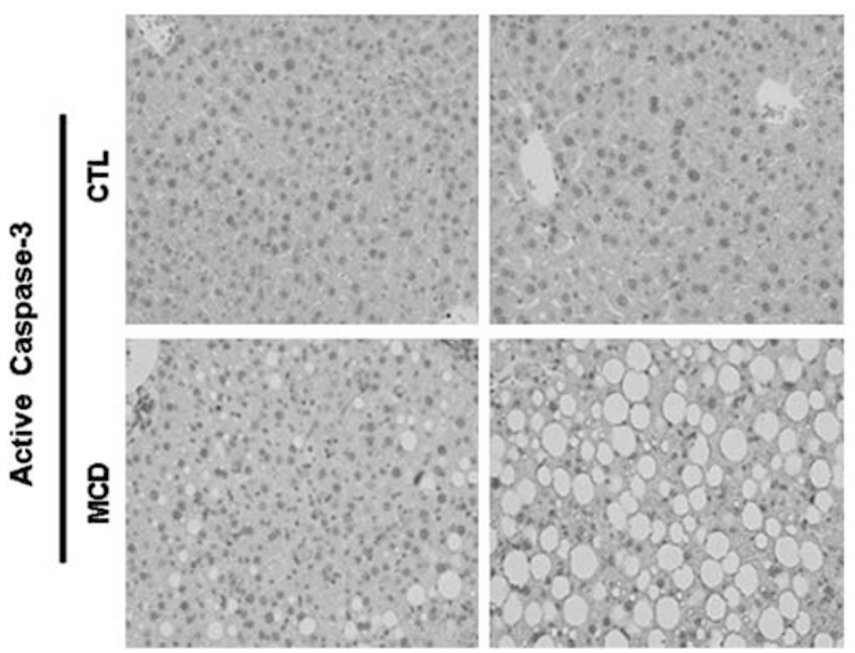

Casp1 - -

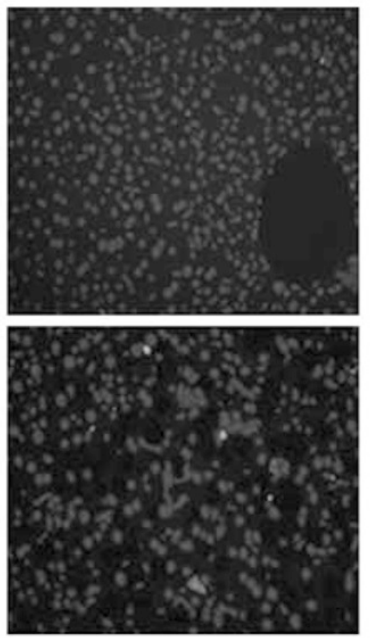

Casp1 $\%$

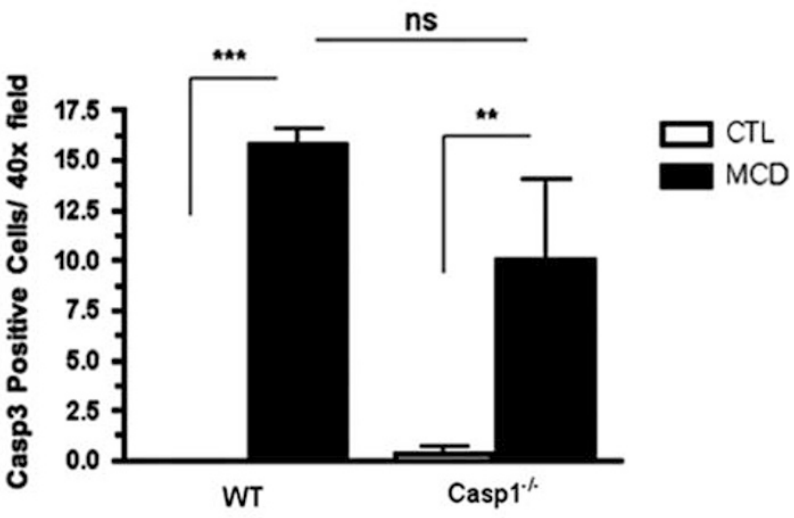

Figure 4 Protection from methionine- and choline-deficient (MCD)-diet induced fibrosis in caspase-1-knockout (Casp $1^{-/-}$) mice is independent of caspase-3 activation and hepatocellular apoptosis. (a, b) Representative microphotographs of terminal deoxynucleotidyl transferase dUTP nick-end labeling (TUNEL) staining, and active caspase-3 immunohistochemistry $(\times 40)$. (c, d) Hepatocyte apoptosis in liver sections was quantified by counting the number of TUNELpositive cells, and cleaved caspase-3-positive cells in five random microscopic fields ( $\times 40$ ). Results are expressed as mean \pm s.e.m. ${ }^{\star} P<0.05$, ${ }^{\star * P}<0.01$, $* * * P<0.001$, compared with controls $(\mathrm{CTL})$.

the fact that the inflammasome is primarily present in monocytes and macrophages, led us to the hypothesis that Kupffer cells, the liver resident macrophages, are the main source for these changes. To test this hypothesis, we next examine the effects of selective depletion of Kupffer cells on caspase-1 expression and MCD-induced liver damage. C57BL/6 WT mice on a 6-week MCD diet were injected twice during the last week of the diet with CLOD or PBS liposomes intravenously. Hepatic steatosis was unchanged between the CLOD- and PBS-treated MCD-fed mice (Figure 5a). Hepatic F4/80 immunostaining demonstrated the effectiveness of CLOD treatment in the depletion of Kupffer cells in the liver (Figure 5a). This was further confirmed by measurement of mRNA levels by RT-PCR of TNF $\alpha$, F4/80 and CD68, which were markedly decreased in the CLOD-treated group com- pared with the PBS-treated MCD-fed mice (Figure 5b). More importantly, caspase-1 protein expression was also significantly reduced in CLOD-treated MCD-fed mice as detected by both immunohistochemistry of liver tissue and immunoblot analysis of whole-liver lysates (Figure 5c-e). These changes were associated with a decrease in the expression of genes involved in fibrogenesis (Figure 5f), as well as a dramatical reduction in collagen deposition in CLOD-treated MCD-fed mice (Figure 5g). These data strongly suggest that Kupffer cells are the main cellular source of active caspase-1 in MCD-diet-induced steatohepatitis.

\section{DISCUSSION}

The principle findings of this study relate to the role of caspase-1 during NASH development. The results demonstrate 
a

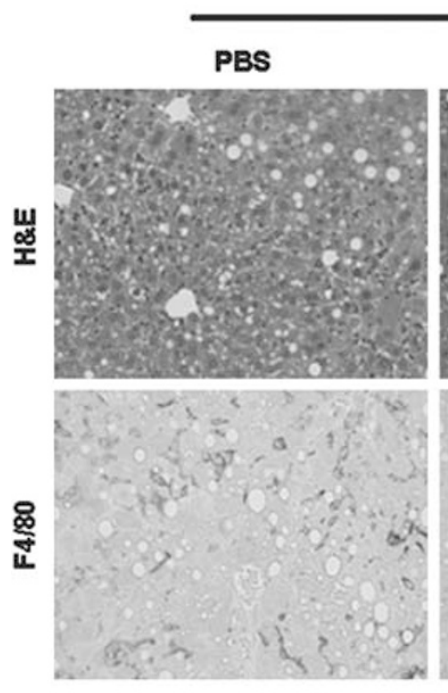

b

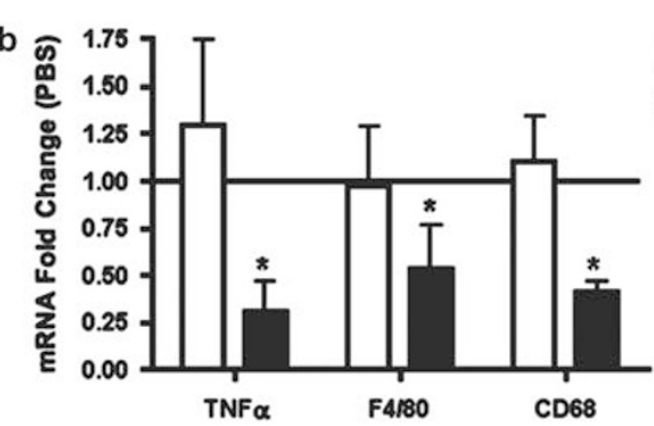

f

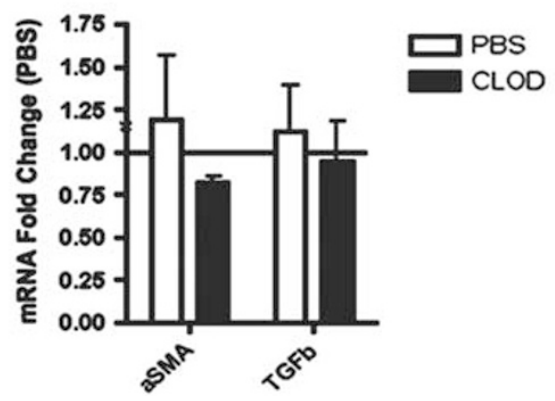

C
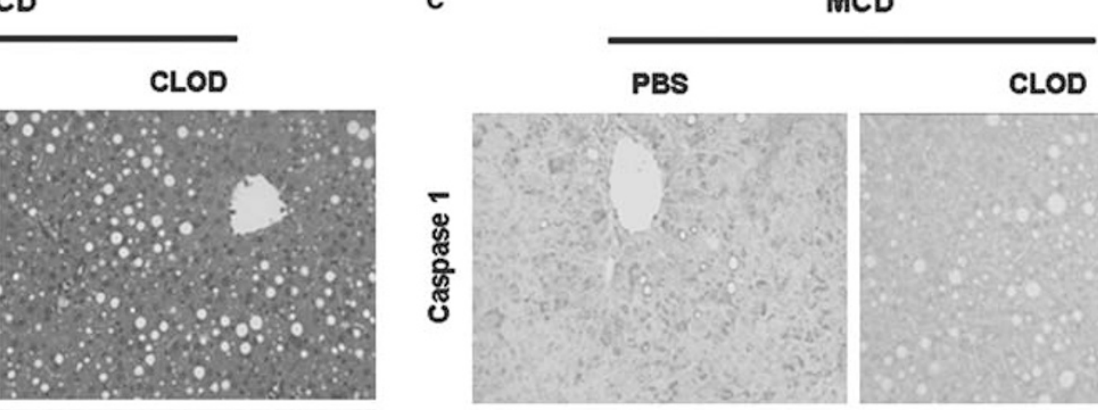

d
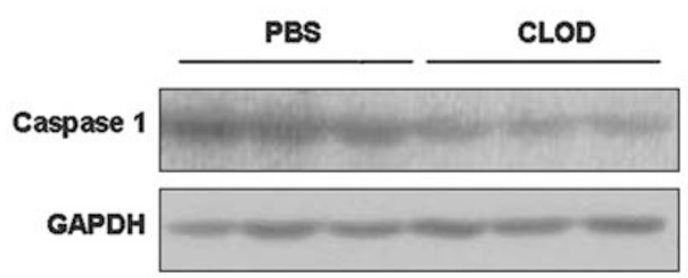

e

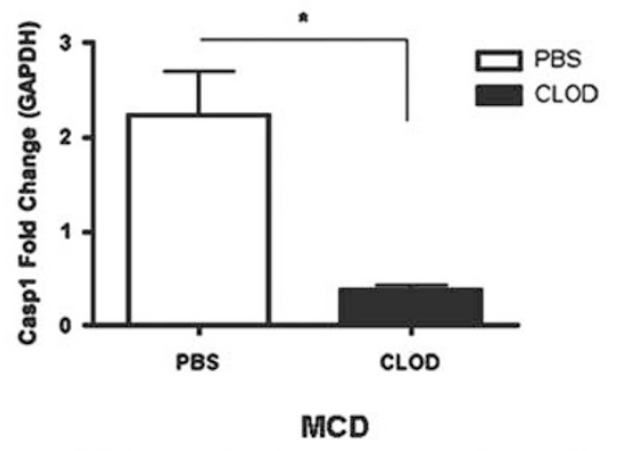

g

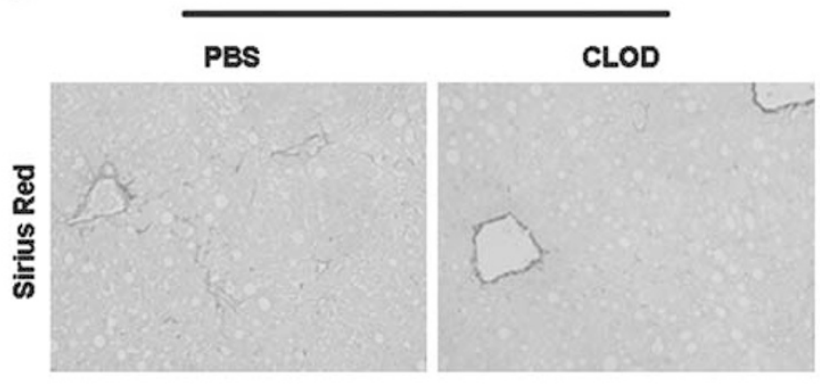

Figure 5 Kupffer cell depletion abrogates caspase-1-mediated diet-induced steatohepatitis. (a) Representative photomicrographs of hematoxylin and eosin (H\&E), and F4/80 immunostaining in the liver of phosphate vehicle (PBS)- $(n=7)$ or clodronate (CLOD)-treated $(n=3)$ MCD-fed mice. (b) RT-PCR analysis of tumor necrosis factor- $\alpha(\mathrm{TNF} \alpha)$ and macrophage markers F4/80 and CD68 expression in the liver of PBS- $(n=7)$ or CLOD-treated ( $n=3)$ MCD-fed mice. (c) Representative photomicrographs of caspase-1 immunostaining on liver tissue from the two groups of mice, and (d, e) western blot analysis of caspase-1 performed on whole-liver tissue lysates from PBS- $(n=7)$ or CLOD-treated $(n=3)$ MCD-fed mice with densitometric analysis. (f) RT-PCR analysis of fibrogenesis genes $\alpha$-smooth muscle actin ( $\alpha$ SMA) and transforming growth factor- $\beta$ (TGF $\beta$ ) expression in the liver of PBS- $(n=5)$ or CLOD-treated ( $n=3$ ) MCD-fed mice. (g) Collagen fibers were stained with Sirius red. Results are expressed as mean \pm s.e.m. ${ }^{*} P<0.05$ compared with controls (CTL).

that NASH induced by MCD feeding is associated with caspase-1 activation in the liver, whereas caspase- 1 suppression is associated with dissociation between hepatic triglyceride accumulation and inflammatory activity, and protects against HSC activation and fibrosis development. These effects were independent of caspase-3 activation and hepatocyte cell death. Furthermore, we identified Kupffer cells as a key cellular source of active caspase-1 in MCD diet-induced steatohepatitis.
Since the original description that caspase- 3 and -7 activation and TUNEL-positive cells are characteristic pathological features in the liver of NASH patients, ${ }^{9}$ a growth of data, mainly from experimental studies, have suggested that caspase activation, mainly caspase-3, is a key process involved in NASH pathogenesis. ${ }^{10}$ As a result, the targeting of caspase activity has gained significant attention for developing of both novel therapeutic as well as diagnostic strategies for NASH patients. A recent pre-clinical study tested a 
pan-caspase inhibitor VX-166 in an animal model of NASH..$^{19}$ Obese leptin receptor-deficient $\mathrm{db} / \mathrm{db}$ mice were fed MCD diet to induce NASH and liver fibrosis. Mice gavaged daily with VX-166 showed a marked reduction in hepatic caspases activity, decreased levels of mature IL-1 $\beta$ and IL-18 in the liver and liver fibrosis.

Caspases belong to a family of highly conserved cysteinedependent aspartate-specific acid proteases that use a cysteine residue as the catalytic nucleophile and share a stringent specificity for cleaving their substrates after aspartic acid residues in target proteins. ${ }^{15}$ They are synthesized as inert zymogens, and upon receiving apoptotic stimuli, cells activate initiator caspases, such as caspase-1, -2, -8, -9 and -10 that, in turn, proteolytically cleave and activate effector caspases, including caspase- $3,-6$ and $-7 .{ }^{16}$ Caspases have been further categorized as either proinflammatory or proapoptotic, depending upon their participation in these cellular programs. The proinflammatory caspases include caspase-1, -11 and -12 in mouse and caspase- $1,-4$ and -5 in human. The relative contribution of pro-apoptotic vs proinflammatory caspases to liver pathology during NASH development as well as in the protective effects of pan-caspase inhibitors remain incompletely understood. Our results confirmed the findings from Witek et al ${ }^{19}$ demonstrating that MCD feeding results in marked caspase-1 activation. Our current data further extend these observations by demonstrating that activated cleaved caspase-1 localized predominantly on non-parenchymal sinusoidal cells in the liver, and to a lesser extent in hepatocytes. Suppression of caspase-1 activation resulted in decreased tissue inflammation despite an increase in triglyceride deposition and hepatic steatosis. Moreover, although as expected after 6 weeks on the MCD diet, WT animals showed a marked increase in the expression of various genes involved in HSC activation and fibrogenesis; these changes were significantly reduced in the Casp1 $1^{-1-}$ mice. More importantly, WT animals on the MCD diet, but not Casp $1^{-1-}$ mice, showed a significant increase in collagen deposition. These changes were independent of hepatocyte caspase-3 activation, hepatocellular injury, as evidenced by ballooning degeneration of hepatocytes and elevation of serum transaminases, and cell death, which occurred to a similar extent in both WT animals and Casp1 $1^{-1-}$ mice on the MCD diet. The precise mechanisms resulting in the catalytic processing of pro-caspase- 1 into its enzymatically active form during NASH development will require further investigation, but multiple processes that are known to induce the assembly of the inflammasome, the caspase-1-activating complex, such as increase in reactive oxygen species production, and lysosomal permeabilization and release of cathepsins into the cytosol are known to be present in both experimental model of NASH as well as humans with this condition. ${ }^{13,28}$

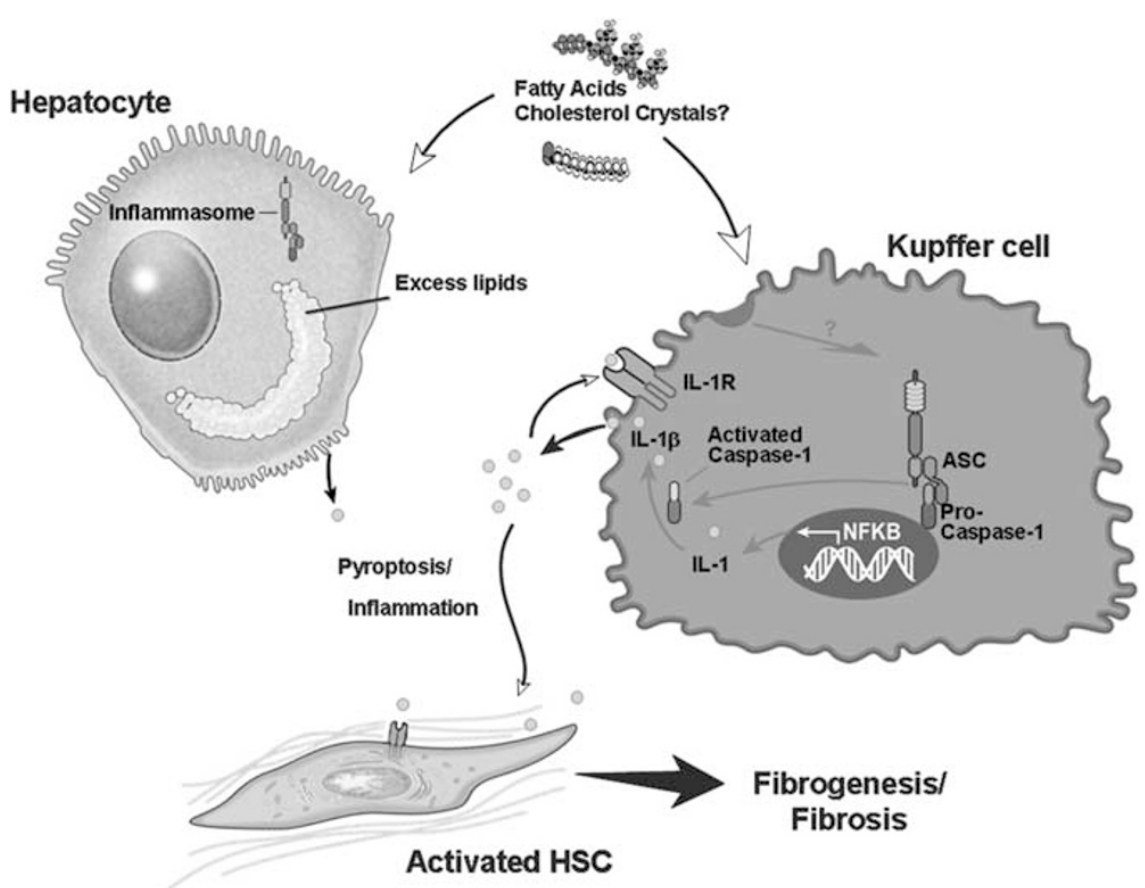

Figure 6 Proposed model for role of inflammasome and caspase-1 activation in tissue damage and fibrosis in steatohepatitis. In the process of nonalcoholic steatohepatitis (NASH) development, different lipotoxic substances, such as cholesterol crystals or free fatty acids, may induce the processing and activation of caspase-1 predominantly in Kupffer cells in the liver, and to a lesser extent in hepatocytes. Hepatic caspase- 1 activation induce cleavage of interleukin (IL)-1 into its mature form IL-1 $\beta$, resulting in increased inflammation and tissue damage, including the activation of hepatic stellate cells. This results in fibrogenesis and fibrosis development. Therapy target at inhibiting the inflammasome or caspase-1 activation may be a novel therapeutic target for treatment of NASH. 
Our key finding of a mixed pattern of cellular expression of caspase-1 in this model of steatohepatitis, in conjunction with the recent report that fatty acids may induce activation of caspase-1 in isolated hepatocytes ${ }^{29}$ led us to further investigate the potential cellular source of this protease. Interestingly, we observed that selective depletion of Kupffer cells by CLOD treatment markedly reduced the protein expression of caspase-1 in the liver of MCD-fed animals, as well as significantly reduced the amount of collagen deposition pro-inflammatory cytokine TNF $\alpha$ in a similar manner to the $\operatorname{Casp1}^{-1-}$ mice on the MCD diet. These results strongly suggest that Kupffer cells are a key cellular source of active caspase-1 in MCD-induced steatohepatitis, which has an important role in the pathogenesis of this model through inflammation and hepatic stellate cell activation and fibrogenesis. Future studies such as those using cell type-specific caspase-1 knock-out mice will be required to further dissect the role of caspase- 1 activation in other cell types in the liver in vivo and their potential distinct role in the pathophysiological changes observed during NASH development.

In summary, the current studies uncover the role of hepatic caspase- 1 activation in experimental NASH. The results support a model in which during the development of NASH, caspase-1 activation in Kupffer cells results in induction of pro-inflammatory signaling and hepatic stellate cell activation, which are then responsible for collagen deposition and fibrosis (Figure 6). These data provide new insights into the pathogenesis of liver damage in NASH and identify potential novel molecular targets for therapeutic intervention in this highly common and potentially serious disease.

\section{ACKNOWLEDGEMENT}

This work was supported by NIH Grants (DK076852) and (DK082451) to AEF.

\section{DISCLOSURE/CONFLICT OF INTEREST}

The authors declare no conflict of interest.

1. Wieckowska A, Feldstein AE. Nonalcoholic fatty liver disease in the pediatric population: a review. Curr Opin Pediatr 2005;17:636-641.

2. Angulo P. Nonalcoholic fatty liver disease. $N$ Engl J Med 2002:346:1221-1231.

3. Browning JD, Szczepaniak LS, Dobbins R, et al. Prevalence of hepatic steatosis in an urban population in the United States: impact of ethnicity. Hepatology 2004;40:1387-1395.

4. Schwimmer JB, Deutsch R, Kahen $T$, et al. Prevalence of fatty liver in children and adolescents. Pediatrics 2006;118:1388-1393.

5. Brunt EM, Neuschwander-Tetri BA, Oliver D, et al. Nonalcoholic steatohepatitis: histologic features and clinical correlations with 30 blinded biopsy specimens. Hum Pathol 2004;35:1070-1082.

6. Adams LA, Lymp JF, St Sauver J, et al. The natural history of nonalcoholic fatty liver disease: a population-based cohort study. Gastroenterology 2005;129:113-121.
7. Matteoni CA, Younossi ZM, Gramlich T, et al. Nonalcoholic fatty liver disease: a spectrum of clinical and pathological severity. Gastroenterology 1999;116:1413-1419.

8. Ekstedt M, Franzen LE, Mathiesen UL, et al. Long-term follow-up of patients with NAFLD and elevated liver enzymes. Hepatology 2006;44:865-873.

9. Feldstein $A E$, Canbay $A$, Angulo $P$, et al. Hepatocyte apoptosis and fas expression are prominent features of human nonalcoholic steatohepatitis. Gastroenterology 2003;125:437-443.

10. Cazanave SC, Gores GJ. Mechanisms and clinical implications of hepatocyte lipoapoptosis. Clin Lipidol 2010;5:71-85.

11. Feldstein A, Gores GJ. Steatohepatitis and apoptosis: therapeutic implications. Am J Gastroenterol 2004;99:1718-1719.

12. Feldstein $A E$, Canbay $A$, Guicciardi $M E$, et al. Diet associated hepatic steatosis sensitizes to Fas mediated liver injury in mice. J Hepatol 2003;39:978-983.

13. Feldstein $A E$, Werneburg NW, Canbay A, et al. Free fatty acids promote hepatic lipotoxicity by stimulating TNF-alpha expression via a lysosomal pathway. Hepatology 2004;40:185-194.

14. Chowdhury I, Tharakan B, Bhat GK. Caspases - an update. Comp Biochem Physiol B Biochem Mol Biol 2008;151:10-27.

15. Li J, Yuan J. Caspases in apoptosis and beyond. Oncogene 2008;27:6194-6206.

16. Weber IT, Fang B, Agniswamy J. Caspases: structure-guided design of drugs to control cell death. Mini Rev Med Chem 2008;8:1154-1162.

17. Anstee QM, Concas $D$, Kudo $H$, et al. Impact of pan-caspase inhibition in animal models of established steatosis and non-alcoholic steatohepatitis. J Hepatol 2010;53:542-550.

18. Barreyro FJ, Holod S, Finocchietto PV, et al. PF-03491390 Pan-caspase inhibitor decreases liver injury and fibrosis in a murine model of nonalcoholic steatohepatitis (NASH). J Hepatol 2010;52:S303.

19. Witek RP, Stone WC, Karaca FG, et al. Pan-caspase inhibitor VX-166 reduces fibrosis in an animal model of nonalcoholic steatohepatitis. Hepatology 2009;50:1421-1430.

20. Kuida K, Lippke JA, Ku G, et al. Altered cytokine export and apoptosis in mice deficient in interleukin-1 beta converting enzyme. Science 1995;267:2000.

21. Li $\mathrm{P}$, Allen $\mathrm{H}$, Banerjee $\mathrm{S}$, et al. Mice deficient in IL-1 beta-converting enzyme are defective in production of mature IL-1 beta and resistant to endotoxic shock. Cell 1995;80:401.

22. Dela Pena A, Leclercq I, Field J, et al. NF-kappaB activation, rather than TNF, mediates hepatic inflammation in a murine dietary model of steatohepatitis. Gastroenterology 2005;129:1663-1674.

23. Den Boer M, Voshol PJ, Kuipers F, et al. Hepatic steatosis: a mediator of the metabolic syndrome. Lessons from animal models. Arterioscl Throm Vas Biol 2004;24:644.

24. London RM, George J. Pathogenesis of NASH: animal models. Clin Liver Disease 2007;11:55-74.

25. Van Rooijen N, Sanders A. Kupffer cell depletion by liposome-delivered drugs: comparative activity of intracellular clodronate, propamidine, and ethylenediaminetetraacetic acid. Hepatology 1996;23:1239-1243.

26. Canbay A, Guicciardi ME, Higuchi $\mathrm{H}$, et al. Cathepsin B inactivation attenuates hepatic injury and fibrosis during cholestasis. J Clin Invest 2003;112:152-159.

27. Kleiner DE, Brunt EM, Van Natta M, et al. Design and validation of a histological scoring system for nonalcoholic fatty liver disease. Hepatology 2005;41:1313-1321.

28. Li Z, Berk M, McIntyre TM, et al. The lysosomal-mitochondrial axis in free fatty acid-induced hepatic lipotoxicity. Hepatology 2008;47: 1495-1503.

29. Csak T, Ganz M, Pespisa J, et al. Fatty acid and endotoxin activate inflammasomes in mouse hepatocytes that release danger signals to stimulate immune cells. Hepatology 2011;54:133-144. 